\title{
Durable and Effectiveness of Immunotherapy in the Treat- ment of Metastatic Gastroesophageal Junction Cancer
}

\section{Carlos Gil Moreira Ferreira, MBBS ${ }^{1}$ (D) Gustavo Bittencourt Camilo ${ }^{2,3^{*}}{ }^{(D)}$, Eleusa Nogueira Dias ${ }^{3}$ (D) Bianca Lopes de Oliveira $^{3}$ (D), Carlos Murilo Schanuel ${ }^{(D)}$, Juliana Abreu de Vasconcellos ${ }^{1}$ (D) and Emílio Campos Augusto Pereira de Assis ${ }^{3}$ (D)}

${ }^{1}$ Department of Oncology, Instituto Oncoclínicas, Rio de Janeiro, Brazil

${ }^{2}$ Department of Radiology, Terezinha de Jesus Hospital and Maternity, Minas Gerais, Brazil

${ }^{3}$ Faculty of Health Sciences and Medicine, SUPREMA, Minas Gerais, Brazil

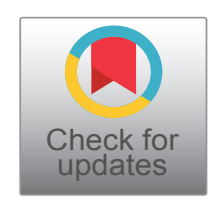

*Corresponding author: Dr. Gustavo Bittencourt Camilo, PhD, Department of Radiology, Terezinha de Jesus Hospital and Maternity; Faculty of Health Sciences and Medicine, SUPREMA, Alameda Salvaterra, 200, Juiz de Fora, Minas Gerais, Brazil, Tel: 21015000

\author{
Keywords \\ Immunotherapy, Medical oncology, Diagnostic imaging, Stom- \\ ach neoplasms
}

\section{Background}

Gastroesophageal junction adenocarcinoma (AJEG) is a neoplasm that is globally prevalent with generally bad clinical outcome [1]. It is estimated that there is an annual incidence of new tumors in the order of 572,034 in the US with about 28,000 new cases and 10,960 deaths attributable to the disease [2,3]. In Brazil, an estimated 11390 new cases, corresponding to 8690 in men and 2077 in women for the year 2020 [4]. About half of the people with cancer of the esophagus or the gastroesophageal junction have metastatic disease at diagnosis, and there are few effective options after the progression of second-line chemotherapy $[5,6]$. Thus, surgery with or without adjuvant or neoadjuvant therapy is the treatment of choice for resectable cases with curative intent [7]. However, in inoperable cases, the conduit to be held is isolated chemotherapy or combined neoadjuvant chemotherapy associated with radiotherapy and is based on Cisplatin, Fluorouracil, Docetaxel, Epirubicin, Irinotecan, Fluoretaacin, Methotrexate, Epirubicin, and Capecitabine [8]. However, some patients do not respond effectively to these procedures. Thus, we adopted a second-line therapy using a targeted approach with immunotherapy based on immunohistochemical tests. Immune checkpoint blocking is a promising treatment strategy for certain cases of advanced cancer, and the immunotherapy strategies that had been used in patients with melanoma, non-small cell lung cancer and urothelial cancer were tested in patients with AJEG [9]. As a result, Pembrolizumab, a programmed cell death protein 1 (PD-1) inhibitor, has recently been approved for metastatic or recurrent JEG that has progressed beyond the second line of systemic therapy and has been used effectively in the present case report [6]. Therefore, it is necessary to find new options for these patients due to high mortality in view of the limitations of first-line treatments.

\section{Case Presentation}

A 72-year-old woman in June 2015 was diagnosed with AJEG G2 (Figure 1 and Figure 2). The oncologist suggested a neoadjuvant chemotherapy scheme with Capecitabine, Oxaliplatin, and Epirubicin (EOX). However, this was not approved because of the cardiotoxicity of Epirubicin since the patient had coronary artery disease and a stent installed. Therefore, neoadjuvant chemotherapy was administered with only Capecit-

Citation: Ferreira CGM, Camilo GB, Dias EN, de Oliveira BL, Schanuel CM, et al. (2020) Durable and Effectiveness of Immunotherapy in the Treatment of Metastatic Gastroesophageal Junction Cancer. Int J Immunol Immunother 7:053. doi.org/10.23937/2378-3672/1410053

Accepted: July 07, 2020: Published: July 09, 2020

Copyright: (c) 2020 Ferreira CGM, et al. This is an open-access article distributed under the terms of the Creative Commons Attribution License, which permits unrestricted use, distribution, and reproduction in any medium, provided the original author and source are credited. 


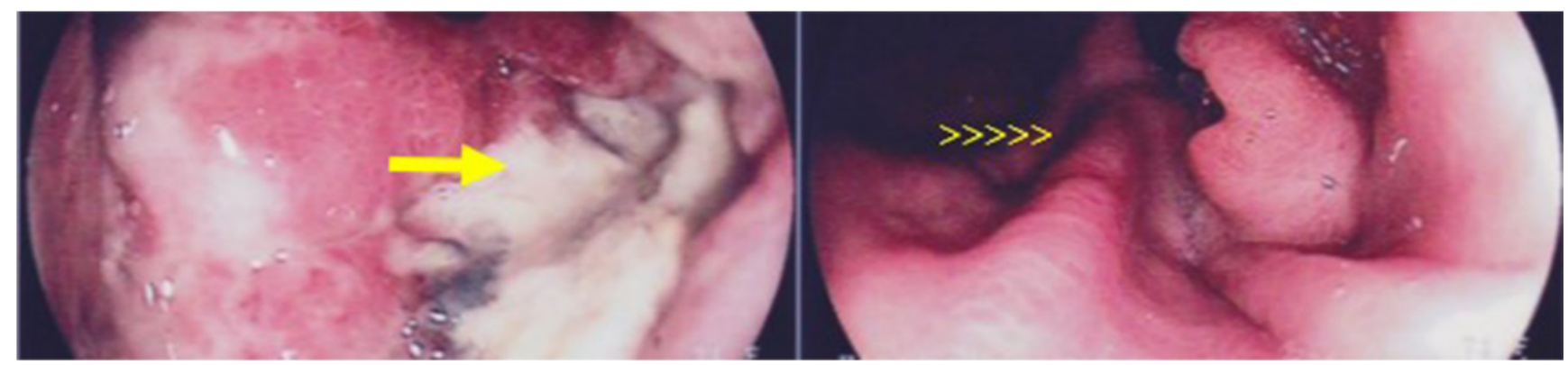

Figure 1: Upper Gastrointestinal Endoscopy showing deep ulceration in the gastric esophagus transition (arrow) and in rear view shows the folds (arrowheads).

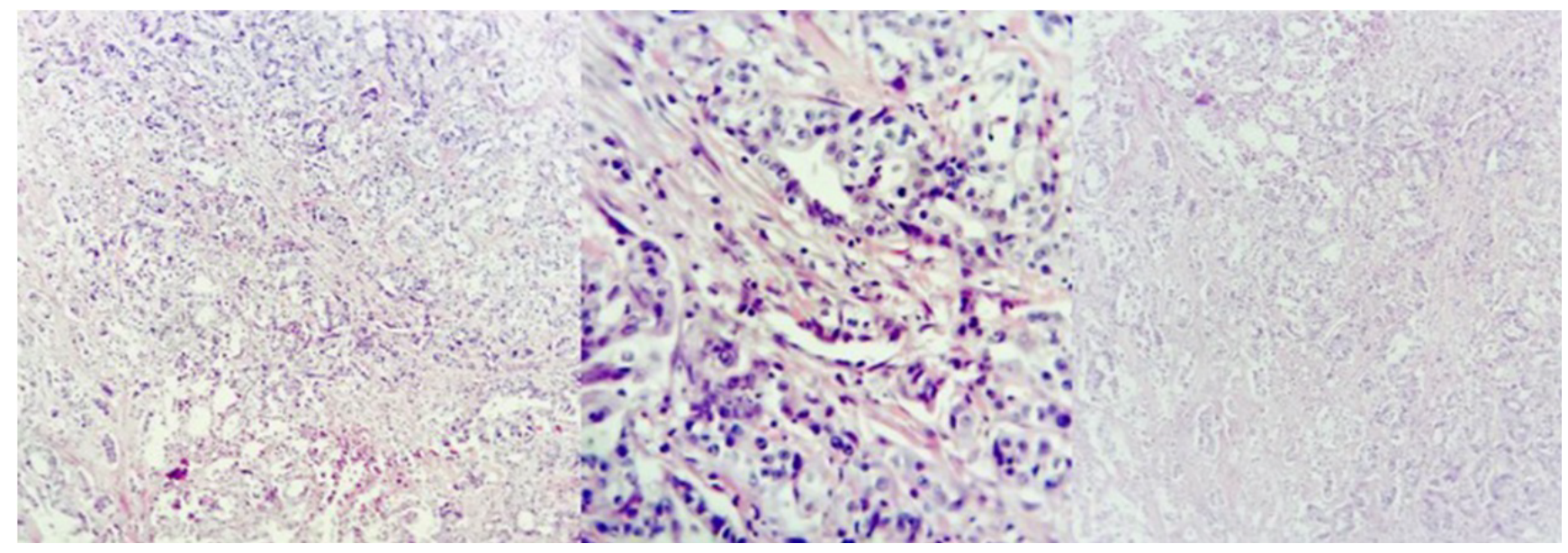

Figure 2: Histopathological slide with H\&E staining, respectively with 40x, 100x and 400x magnifications, obtained in Leica dm 500 microscope and Leica icc50 hd camera. It's possible to observe a typical glands infiltrating the stroma with mild desmoplasia.

abine plus Oxaliplatin. Unfortunately, she had a severe infusion reaction with edema of the glottis during the second application of Oxaliplatin, preventing its further use. This was then followed by therapy with Capecitabine alone, because of which the patient developed extensive gastrointestinal toxicity requiring prolonged hospitalization, experienced significant weight loss (20 $\mathrm{kg}$ in 33 days) and underwent a gastrostomy. We chose to stop the neoadjuvant chemotherapy, and during the months of recovery, she had progression of local disease. In November 2015, the patient underwent gastroesophagectomy (total esophagectomy with extensive gastrectomy and creation of a neoesophagus with great gastric curvature) with an uneventful postoperative period. She had six months without any cancer treatment. In May 2016, a positron-emission tomography and computed tomography (PET-CT) performed on the patient identified nodal, liver, and peritoneal progression and prompted an examination with Personna ${ }^{\circledR}$ Onco, a genetic analysis tool that uses sequencing technology for analysis of multigenic next-generation sequencing (NGS). This examination discarded HER-2 changes and identified the PI3Kca mutation.

In June 2016, a new weekly chemotherapy regimen with Paclitaxel was begun. After 3 cycles, a PET-CT performed in January 2017 to evaluate response to treat- ment, found increased lymph nodes and liver subcapsular nodules in segment $\mathrm{VI}$, suggesting progressing disease (PD) compared to the PET-CT in September 2016. Treatment with isolated Ramucirumab was started. In March 2017, Magnetic Resonance Imaging (MRI) showed progressive worsening nodal and liver PD (Figure 3 and Figure 4). Without the expected response to Ramucirumab, in May 2017, we changed the treatment line to Irinotecan alone. A PET-CT performed in June 2017 showed a favorable tumor response; however, partial treatment was instituted (dimensions of increased peritoneal thickening in iliac fossa were related to the increase in necrotic component and not to disease progression). Once isolated, the patient continued Irinotecan for 10 biweekly applications. In September 2017, an MRI revealed a liver nodule in the caudal aspect of segment IV with hypermetabolism signals, infectious and inflammatory changes involving the gallbladder and the liver parenchyma, and increased volume of the right ovary, which had a heterogeneous appearance. After 10 days, ultrasonography showed signs of cholecystitis and compression of the cystic duct by the hepatic hilum lymph node. During this period, the patient underwent microsatellite instability testing noting high instability (favorably to treatment with immunotherapy - being suggested for Pembrolizumab use). However, because immunotherapy treatment can only be initiated in the 


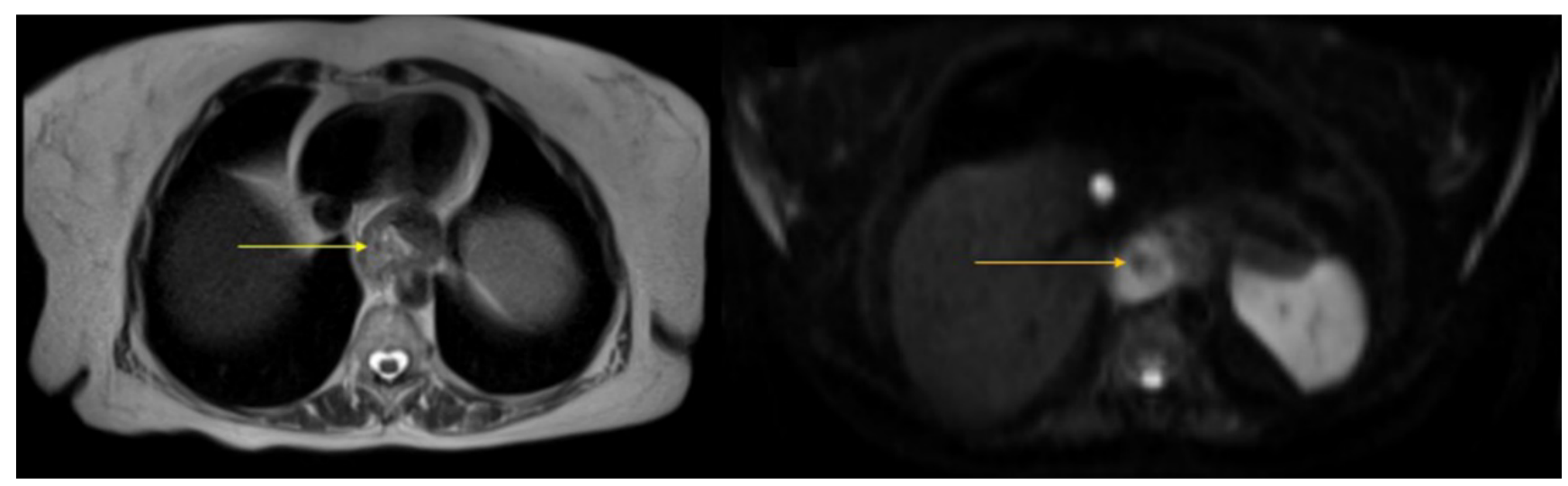

Figure 3: MRI showing an irregular parietal area with discharge at T2 (white arrow) and restriction to diffusion (yellow arrow) at DWI in the herniated gastric esophagus transition.

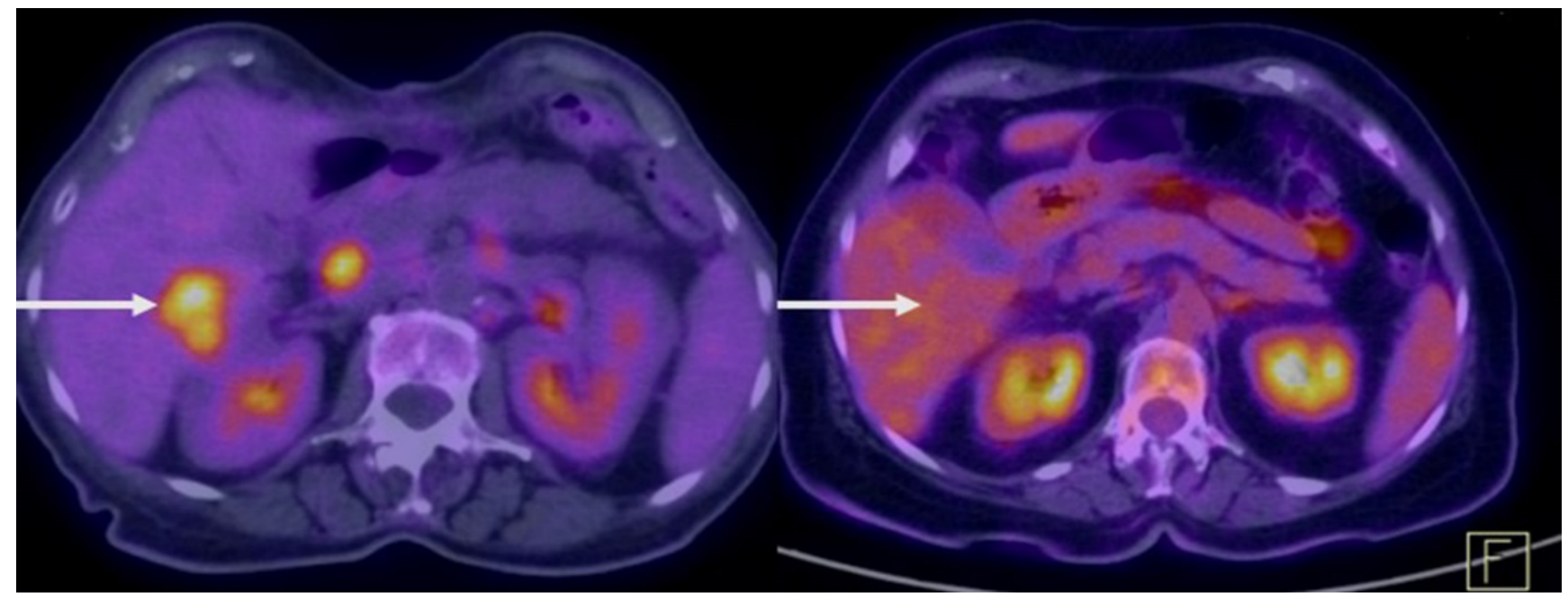

Figure 4: PET-CT in 2017 demonstrating liver nodular lesion in a study carried out immediately before the use of Pembrolizumab suggestive of a secondary implant and in the left at the same level, no longer showing the lesion after using Pembrolizumab, suggestive of complete regression in 2018.

absence of inflammatory processes, a cholecystectomy was required. While awaiting surgery, the patient developed jaundice. Therefore, prosthesis installation was required in the common bile duct by Endoscopic Retrograde Cholangiopancreatography (ERCP), and during the procedure, we opted for permanent metal prosthesis. In September 2017, the patient underwent a cholecystectomy.

In October 2017, after an uneventful postoperative period, the first cycle of immunotherapy was administered with Pembrolizumab $200 \mathrm{mg}$ every 3 weeks. The patient remained in this regimen until revaluation in February 2018 without any relevant side effects. A PET-CT performed in February 2018 demonstrated a set of evolutionary findings suggesting favorable tumor response to treatment. A comparative analysis with the previous PET-CT in September 2017 had not more showed/shown lymphadenopathy in the porto-caval region and not revealed a significant increase of the radiotracer in peritoneal findings and a decrease in attenuation coefficients, suggesting a predominance of the necrotic component (adnexal right, just a capsular in liv- er segment VI, the hepatic hilum, the mesentery at the level of the right iliac fossa, left pre-sacral, and sigmoid handle inseparable). Thus, it was established that there was a favorable response by the drug.

Due to an excellent therapeutic response, the patient was maintained on Pembrolizumab every 3 weeks for another 4 months. A PET-CT in July 2018 not showed the small nodular lesion in liver segment VIII on a comparative analysis with the study from February 2018 and continues ever since (Figure 4).

\section{Discussion}

The immune system has been considered a key factor responsible for monitoring and combating malignant tumors [10,11]. Malignant cells often have genetic and/ or epigenetic alterations that can lead to the synthesis of neoantigens recognizable as "non-self" by the host immune system [12].

The manipulation of the immune system through immunotherapy is possibly a paradigm shift in cancer therapy. For this to be effective, one must consider both components of an immune response to an action on 
immune effector cytotoxic as the action on the mechanisms of tolerance. The knowledge that the immunological status is different for each tumor is a prerequisite for proposing appropriate immune therapies $[13,14]$.

In 2015, Pembrolizumab (Keytruda`, MK3475), humanized immunoglobulin $\mathrm{G}_{4}(\lg \mathrm{G} 4)$, was approved for treatment of refractory melanoma by the Food and Drug Administration (FDA) and the European Medicines Agency (EMA) [15]. More recently, Pembrolizumab was approved by the FDA, EMA, and the Japanese Pharmaceuticals and Medical Devices Agency to treat patients with high levels of programmed death-ligand 1 (PD-L1) $(\geq 50 \%$ ) without chemotherapy expression and nonsmall cell lung cancer, without the EGFR mutation or rearrangement of $A L K$, due to efficacy in patients with tumors with high PD-L1 expression [16].

This drug is one humanized IgG4 antibody isotype that blocks a protective mechanism of cancer cells and allows the immune system to destroy these cancer cells. It targets the PD-1 receptor located on lymphocytes. This receiver, which is typically responsible for preventing the immune system from attacking the body's own tissues, is recognized as immune checkpoint $[17,18]$. The PD-1 is a transmembrane regulatory protein found on the surface of the effector cells of the immune system, such as activated T-cells, B-cells, and myeloid cells, to modulate their activation or inhibition. Under physiological conditions, the interaction between PD-L1 and PD-1 promotes the regulation of the immune response down resulting in negative feedback inhibitory counter $[19,20]$. However, patients who present with high microsatellite instability (MSI-H) have incompatible repair deficient tumors (MMRD), which are highly infiltrated tumors. In this group of individuals, a large number of cytotoxic activated CD8-positive T-cells and checkpoints regulated with PD-1 and/or PD-L1 are found [8,9]. Thus, when expressed on the surface of tumor cells, PD-L1 ligand allows avoidance of immune response to the tumor [21] leading to a depletion of cytotoxic T-lymphocytes that can remain in a state of anergy [22] damaging the immune system's ability to combat malignant cells. Thus, when the PD-1 on the T-cell binds to the PD-L1 in the tumor cells, the T-cell is deactivated, allowing the cancer cell to avoid the immune attack. Therefore, Pembrolizumab aims to inhibit the complex PD-1/PD-L1 by binding to PD-1, allowing the T-cell to remain active and coordinate an attack. However, Wang, et al. speculate that patients with AJEG whose tumor has high levels of PD-L1 expression or with microsatellite instability may be more suitable for anti-PD-1/PD-L1 treatment [23].

Many cancers produce proteins that bind to PD-1, blocking the body's ability to inhibit cancer in isolation $[16,17]$. Inhibition of DP-1 in lymphocytes avoids this process, allowing the immune system to reach and destroy the cancerous cells [24]. Nevertheless, this same mechanism also allows the immune system to attack the body itself and checkpoint inhibitors, such as Pembrolizumab, resulting in immune dysfunction as a side effect of its use. Hypothyroidism (RR 7.73, 95\% Cl: 3.86$15.49, \mathrm{p}<0.001$ ); hyperthyroidism (RR 5.09, 95\% Cl 2.36-10.97, p < 0.001), uveitis and "dry eye", diarrhea, colitis or hepatitis $[18,25-27]$ are the main side effects of this drug.

\section{Conclusion}

Finally, the patient with microsatellite instability was heavily pretreated with chemotherapy and second-line therapy, not obtaining the desired result, like most patients with AJEG. This results in a high mortality rate with limited treatment options and poor prognosis. The current results suggest that pembrolizumab offers a new and promising treatment option for the subgroup of patients with high microsatellite instability [28]. However, more randomized clinical studies and large trials are needed to confirm our findings especially in these sub-patients.

\section{Summary Points}

- Gastroesophageal junction adenocarcinoma (AJEG) is a neoplasm that is globally prevalent with generally bad clinical outcome.

- There are limited treatment options and poor prognosis for these patients.

- Pembrolizumab, a programmed cell death protein 1 (PD-1) inhibitor, has recently been approved for metastatic or recurrent JEG that has progressed beyond the second line of systemic therapy to the subgroup of patients with high microsatellite instability.

- Pembrolizumab was effective in a heavily-pretreated patient with high microsatellite instability.

\section{Financial \& Competing Interests Disclosure}

The authors have no relevant affiliations or financial involvement with any organization or entity with a financial interest in or financial conflict with the subject matter or materials discussed in the manuscript. This includes employment, consultancies, honoraria, stock owner ship or options, expert testimony, grants or patents received or pending, or royalties. No writing assistance was utilized in the production of this manuscript.

\section{Informed Consente Disclosure}

The authors state that they have obtained verbal and written informed consent from the patient/patients for the inclusion of their medical and treatment history within this case report.

\section{References}

1. MR Openshaw, Richards CJ, Guttery DS, JA Shaw, Thomas AL (2017) The genetics of gastroesophageal adenocarcinoma and the use of cell free circulating DNA for detection and monitoring disease. Expert Rev Mol Diagn 17: 459-470. 
2. Siegel RL, Miller KD, Jemal A (2020) Cancer Statistics. J Cancer Clin 67: 7-30.

3. Globocan (2018) Global cancer observatory.

4. (2020) National Cancer Institute. Types of cancer.

5. Janmaat VT, Steyerberg EW, Van der Gaast The, Mathijssen RH, Bruno MJ, et al. (2017) Palliative chemotherapy and targeted therapies for esophageal and gastroesophageal junction cancer. Cochrane Database Syst Rev 28: 11.

6. Cohen IN, Strong VE, Janjigian YY (2018) Checkpoint blockade in esophagogastric cancer. J Surg Oncol 118: 777-857.

7. Heger P, Blank S, Goossen KH, Nienhuser, Diener MK (2019) Thoraco abdominal versus transhiatal surgical approaches for adenocarcinoma of the esophagogastric junction-asystematic review and meta-analysis. Langenbecks Arch Surg 404: 103-113.

8. Wang C, Song DJ, Xu ZL, Xie SP, Hu JH (2017) A network meta-analysis of the short-term efficacy of chemotherapy regimens based on five cisplatin and fluorouracilis esophagogastric junctional adenocarcinoma. Exp Mol Med 49: e383.

9. Goode EF, Smyth EC (2016) Immunotherapy of gastroesophageal cancer. J Clin Med 5: 84.

10. Finn OJ (2012) Immuno-oncology: Understanding the function and dysfunction of the immune system in cancer. Ann Oncol 23: 86-89.

11. Armand $P$ (2015) Immune checkpoint blockade in hematologic malignancies. Blood 125: 3393-3400.

12. The Bylicki, N Paleiron, Rousseau-L Bussac, Chouaid C (2018) New PDL1 inhibitors for non-small cell lung cancer: Focus on pembrolizumab. Onco Ther Targets 16: 40514064.

13. Nayama M, Collinet $P$, Salzet $M$, Vinatier D (2016) Immunological aspects of ovarian cancer: Therapeutic perspectives. J Obstet Gynecol Reprod Biol 45: 1020-1036.

14. Gilligan AM, Skrepnek GH (2014) Determinants of life expectancy in the Eastern mediterranean region. Health Policy Plan.

15. Amy Barone, Maitreyee Hazarika, Marc R Theoret, Pallavi Mishra-Kalyani, Huanyu Chen, et al. (2017) FDA approval summary: Pembrolizumab for the treatment of Patients with unresectable or metastatic melanoma. Clin Cancer Res 23: 5661-5665.

16. Leena Gandhi, Ani Balmanoukian, Rina Hui, Omid Hamid, Naiyer A Rizvi, et al. (2014) CT105 Abstract: MK-3475 (an-
ti-PD-1 monoclonal antibody) for non-small cell lung cancer (NSCLC): Antitumor activity and association with tumor PDL1 expression. Cancer Res 74: 105.

17. Syn NL, Teng MWL, TSK Mok, Soo RA (2017) De-novo and resistence to anquired immune checkpoint targeting. The Lancet Oncology.

18. Buque A, Bloy N, Aranda F (2015) Trial Watch: Monoclonal immunomodulatory antibodies for oncological indications. Oncoimmunology 4.

19. Oiseth S, Aziz M (2017) Cancerimmunotherapy: A brief review of the history, possibilities, and challenges ahead. $J$ Cancer Metastasis Treatment 3: 250-261.

20. Chen L, X Han (2015) Anti-PD-1 / PD-L1 therapy of human cancer: Past, present, and future. J Clin Invest 125: 33843391.

21. Taieb J, Moehler M, N Boku, JA Ajani, Yanez Ruiz E (2018) Evolution of checkpoint inhibitors for the treatment of metastatic gastric cancers: Current status and future perspectives. Cancer Treat Rev 66: 104-113.

22. de Guillebon E, Roussille P, Frouin E, Tougeron D (2015) Antiprogram death-1/antiprogramdeath-ligand 1 in digestive cancers. World J Gastrointest Oncol 7: 95-101.

23. Wang BC, Zhang ZJ, Fu C, Wang C (2019) Efficacy and Safety of anti-PD-1/PD-L1 Agents vs chemotherapy in patients with gastricor gastroesophageal junction cancer: Systematic review and meta-analysis. Medicine (Baltimore) 98: e18054.

24. Pardoll DM (2012) The blockade of immune checkpoints in cancer immunotherapy. Nate Rev Cancer 12: 252-264.

25. Su Q, Zhang XC, Wang DY, Zhang HR, Zhu C, et al. (2018) The risk of immune-related endocrine disorders associated with anti-PD-1 inhibitors therapy for solid tumors: A systematic review and meta-analysis. Int Immunopharmacol 59: 328-338.

26. Dalvin LA, Shields CL, Orloff M, Sato T, Shields JA (2018) Checkpoint immune inhibitor therapy: Systemic ophthalmic indications and side effects. Retina 38: 1063-1078.

27. Karamchandani DM, Chetty R (2018) Immune checkpoint inhibitor-induced gastrointestinal injury and hepatic: Pathologists' perspective. J Clin Pathol 71: 665-671.

28. Shitara K, M Ozguroglu, Bang YJ, Bartolomeo DiM, Mandala M, et al. (2018) Pembrolizumab versus paclitaxel for previously treated, advanced gastricor gastro-oesophageal junction cancer: A randomized, open-label, controlled, phase 3 trial. Lancet 392: 123-133. 